\title{
Preparation and Characterization of a Standardized Anticonvulsant Ginseng Rb Extract from Panax quinquefolius L.
}

\author{
Zhizhen Zhang ${ }^{1,2}$ and Xiao-Yuan Lian*,3
}

${ }^{1}$ National Center for Pharmaceutical Crops, College of Forestry and Agriculture, Stephen F. Austin State University,
Nacogdoches, Texas 75961, USA; ${ }^{2}$ Novobiotic Pharmaceuticals, Cambridge, Massachusetts 02138, USA; ${ }^{3}$ College of
Pharmaceutical Sciences, Zhejiang University, Hangzhou 310058, People's Republic of China

\begin{abstract}
A ginseng $\mathrm{Rb}$ extract (GRbE) containing three major ingredients of ginsenoside $R b_{1}\left(G-R b_{1}\right)$, ginsenoside $R b_{3}$ $\left(\mathrm{G}-\mathrm{Rb}_{3}\right)$ and ginsenoside $\mathrm{Rd}(\mathrm{G}-\mathrm{Rd})$ has been shown to have anticonvulsant and neuroprotective activity. As such, sufficient characterization and standardization of this active GRbE are demanded to facilitate an ongoing preclinical investigation on its potential for the treatment of epilepsy. In the investigation, the methods for generating the active GRbE and isolating $\mathrm{G}-\mathrm{Rb}_{1}, \mathrm{G}-\mathrm{Rb}_{3}$, and G-Rd in large scale from Panax quinquefolius are described. The chemical profile of GRbE was characterized by identifying the structure of the individual ingredients using NMR, and the concentration of individual ingredients was determined using HPLC. This study demonstrates the application of the established methods for reproducibly generating anticonvulsant GRbE, which is enriched in five panaxadiol glycosides of $G-R b_{1}$, ginsenoside $R b_{2}$ $\left(\mathrm{G}-\mathrm{Rb}_{2}\right), \mathrm{G}-\mathrm{Rb} \mathrm{b}_{3}$, ginsenoside $\mathrm{Rc}(\mathrm{G}-\mathrm{Rc})$, and G-Rd with total ginsenosides over $90 \%$, and for purifying $\mathrm{G}-\mathrm{Rb}_{1}, \mathrm{G}-\mathrm{Gb}_{3}$ and G-Rd with purity of $97.9 \%, 96.6 \%$ and $98.6 \%$, respectively.
\end{abstract}

Keywords: Anticonvulsant ginseng $\mathrm{Rb}$ extract, ginsenosides, preparation, characterization, standardization, Panax quinquefolius.

\section{INTRODUCTION}

Ginseng (Panax L.) has been widely used as tonic and restorative agent by Asians for 2,000 years, and is currently a component in numerous herbal dietary supplements used regularly by about 6 million Americans [1]. More than ten species of ginseng reported worldwide are available and used as medicinal resources, but the two most commonly used species are Panax ginseng C.A. Mey. (Asian ginseng) and Panax quinquefolius L. (American ginseng). Both Asian and American ginsengs have been cultivated as pharmaceutical crops for the production of Standardized Therapeutic Extracts (STEs) used in many ginseng products. Although some other constituents of ginseng may have some activity, it is well known that ginsenosides are the major bioactive compounds mainly which are responsible for the effects ascribed to ginseng [2]. To date, over 100 ginsenosides have been isolated and identified from Panax [3-5], and most of them belong to two types of ginsenosides: the panaxadiols (e.g., ginsenosides $\mathrm{Rb}_{1}, \mathrm{Rb}_{2}, \mathrm{Rb}_{3}, \mathrm{Rc}, \mathrm{Rd}, \mathrm{Rg}_{3}, \mathrm{Rh}_{2}$, and $\mathrm{Rh}_{3}$ ) and the panaxatriols (e.g., ginsenosides Re, Rf, $\mathrm{Rg}_{1}, \mathrm{Rg}_{2}$, and $\mathrm{Rh}_{1}$ ). Pharmacological effects attributes to ginsenosides have been shown in the central nervous system, the cardiovascular system, the endocrine system, and the immune system [2].

Recently, a partially purified ginseng Rb extract (GRbE), which is riched in panaxadiol ginsenosides including three major ingredients of ginsenoside $\mathrm{Rb}_{1}\left(\mathrm{G}-\mathrm{Rb}_{1}\right)$, ginsenoside $\mathrm{Rb}_{3}\left(\mathrm{G}-\mathrm{Rb}_{3}\right)$, and ginsenoside $\mathrm{Rd}(\mathrm{G}-\mathrm{Rd})$, has shown signifi-

*Address correspondence to this author at the College of Pharmaceutical Sciences, Zhejiang University, Hangzhou 310058, People's Republic of China; Tel/Fax: 01186-571-8820-8432; E-mail: xylian@zju.edu.cn cant anticonvulsant effect and neuroprotective activity in animal models induced by pilocarpine, kainic acid, pentylenetetrazole, and 3-nitropropionic acid [6-8]. Interestingly, the activities of this GRbE were stronger than those of total ginsenosides (include panaxadiols and panaxatriols) and individual panaxadiols. As such, quality control of this active GRbE is required to facilitate an ongoing preclinical investigation on its potential for the treatment of epilepsy. The aim of present study is to develop methods for preparing and standardizing the active GRbE including the three major ginsenosides $\mathrm{G}-\mathrm{Rb}_{1}, \mathrm{G}-\mathrm{Rb}_{3}$, and G$\mathrm{Rd}$ from $P$. quinquefolius.

\section{MATERIALS AND METHODOLOGY}

\section{Reagents and Materials}

Octadecyl-functionalized silica gel (ODS) and silica gel (60-200 mesh, 60尺) were purchased from Sigma-Aldrich (St. Louis, MO) and used for column chromatography. Reference ginsenosides $\mathrm{Rb}_{1}, \mathrm{Rb}_{2}, \mathrm{Rb}_{3}, \mathrm{Rc}$, and $\mathrm{Rd}$ were isolated by preparative and analytic HPLC from GRbE. The structures (Fig. 1) of these ginsenosides were determined by extensive NMR analysis with the purity of $98.5 \%-99.3 \%$ determined by HPLC and were used as reference compounds. All solvents used for extract and column elution (ACS grade) and HPLC analysis (HPLC grade) were purchased from EMD Chemicals Inc. (Gibbstown, New Jersey).

Five batches of the roots of $P$. quinquefolius (American ginseng, termed samples A-E) were purchased from different herbal product stores. Samples A and B were purchased from Yan on Ginseng Co. (Houston, USA), samples C and D 


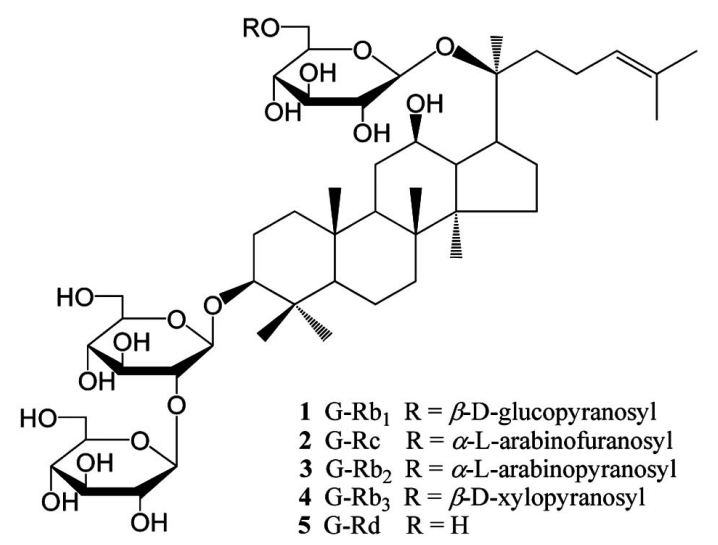

Fig. (1). Structure of five ginsenosides.

were obtained from Zhangtongtai Chinese Medicine Pharmacy (Hangzhou, China), and sample $\mathrm{E}$ was purchased from Qingshanlu Chinese Medicine Pharmacy (Nanchang, China).

\section{Apparatus}

NMR experiments were performed on a Bruker $600 \mathrm{MHz}$ NMR instrument. HPLC analysis was performed on a Hypersil ODS column (column A, $250 \times 4.6 \mathrm{~mm}, 5 \mu \mathrm{m}$, Supelco) using a Hewlett Packard Series 1100 with a HP 1100 diode array detector. Preparative HPLC was performed with an Acuflow Series III pump connected with an Acutect 500 UV/VIS detector using an Econosil ODS column (column B, $250 \times 22 \mathrm{~mm}, 10 \mu \mathrm{m}$, Alltech).

\section{Preparation of Ginseng Rb Extract}

The roots of American ginseng (sample A, $600 \mathrm{~g}$ ) were ground to a coarse powder and percolated with methanol for three times (each $4000 \mathrm{~mL}$ ). Combined methanol solution was concentrated under vacuum to give methanol extract $(60.8 \mathrm{~g})$. This extract was dissolved in 50\% methanol (200 $\mathrm{mL})$ and then chromatographed on a column $(600 \times 70 \mathrm{~mm})$ of octadecyl-functionalized silica gel (ODS, 1,000 g) eluting with $50 \%$ and $70 \%$ methanol (each, $3,500 \mathrm{~mL}$ ), respectively. Each $70 \%$ methanol fraction $(200 \mathrm{~mL})$ collected from the column was analyzed by HPLC. The fractions 20-33 $(4,000-6,600 \mathrm{~mL})$ were combined, evaporated under a vacuum, and then freeze-dried to give fraction A $(11.6 \mathrm{~g})$. Fraction A was further purified using a column $(500 \times 45 \mathrm{~mm})$ of silica gel $(500 \mathrm{~g})$ eluting with chloroform- $\mathrm{MeOH}(3: 1,1,500$ $\mathrm{mL})$ and then chloroform-MeOH $(2: 1,1,500 \mathrm{~mL})$. The chloroform-MeOH (2:1) elution was evaporated under a vacuum to give the ginseng $\mathrm{Rb}$ extract A (GRbE A, $9.6 \mathrm{~g}$ ). Using the same procedure, GRbE $A_{1}(9.2 \mathrm{~g})$ and $\operatorname{GRbE~A}_{2}(9.3 \mathrm{~g})$ were repeatedly obtained from sample $\mathrm{A}$, and $\mathrm{GRbE} \mathrm{B}(10.8 \mathrm{~g})$, GRbE C ( $7.2 \mathrm{~g})$, GRbE D (11.7 g), and GRbE E (7.8 g) were generated from the samples B-E (each $600 \mathrm{~g}$ ), respectively.

\section{Investigation of the Chemical Composition of the Ginseng Rb Extract}

The GRbE A ( $1.0 \mathrm{~g})$ was further separated by preparative HPLC using UV detection at $203 \mathrm{~nm}$, flow rate at 5.0 $\mathrm{mL} / \mathrm{min}$ and mobile phase of $\mathrm{CNCH}_{3} / \mathrm{H}_{2} \mathrm{O}, 40 / 60$ ) to obtain compounds 1 (236 mg, $\left.\mathrm{t}_{R} 35 \mathrm{~min}\right), 2\left(38 \mathrm{mg}, \mathrm{t}_{R} 46 \mathrm{~min}\right)$, a mixture of $\mathbf{3}$ and $\mathbf{4}\left(490 \mathrm{mg}, \mathrm{t}_{R} 55 \mathrm{~min}\right)$, and $\mathbf{5}\left(136 \mathrm{mg}, \mathrm{t}_{R} 76\right.$ min). The mixture of $\mathbf{3}$ and $\mathbf{4}$ was further separated by HPLC using a Hypersil ODS column $(250 \times 4.6 \mathrm{~mm}, 5 \mu \mathrm{m}$; detection: $203 \mathrm{~nm}$; flow rate: $1.5 \mathrm{~mL} / \mathrm{min}$; flow phase: $\left.\mathrm{CNCH}_{3} / \mathrm{H}_{2} \mathrm{O}, 32 / 68\right)$ to afford $3\left(120 \mathrm{mg}, \mathrm{t}_{R} 16.1 \mathrm{~min}\right)$ and 4 (350 mg, $\left.\mathrm{t}_{R} 18.1 \mathrm{~min}\right)$.

\section{Structure and Purity Determination of Compounds 1-5}

The structures of compounds 1-5 were determined on the basis of extensive NMR analysis $\left({ }^{1} \mathrm{H}\right.$ and ${ }^{13} \mathrm{C}$ NMR, ${ }^{1} \mathrm{H}-{ }^{1} \mathrm{H}$ COSY, HMQC, and HMBC experiments) and in comparison with the reported NMR data [9-11]. The purity of $\mathbf{1 - 5}$ was determined by HPLC.

\section{Isolation and Purification of Individual Ginsenosides $R b_{1}$, $R b_{3}$, and $R d$ in Large Scale}

GRbE $(18.0 \mathrm{~g})$ was dissolved in $50 \%$ methanol $(100 \mathrm{~mL})$ and then applied on a column $(600 \times 70 \mathrm{~mm})$ of ODS $(1,000$ g) eluting with $5000 \mathrm{~mL}$ of $65 \%$ methanol. Each fraction $(100 \mathrm{~mL})$ collected from the column was analyzed by HPLC. The fractions containing single compound were combined, evaporated under vacuum, and then freeze-dried to give G$\mathrm{Rb}_{1}$ (4.12 g, fractions 9-15, 900-1,500 mL), G-Rb 3 (3.56 g, fractions $25-30,2,500-3,000 \mathrm{~mL})$, and G-Rd $(2.05 \mathrm{~g}$, fractions 41-46, 4,100-4,600 mL).

\section{Preparation of Reference Ginsenosides and Samples Solu- tion}

Each reference ginsenoside $(\sim 5.0 \mathrm{mg})$ was accurately weighted into a $5.0 \mathrm{~mL}$ volumetric flask and dissolved in a mixture of acetonitrile/water (40:60) to make a stock solution of $1.0 \mathrm{mg} / \mathrm{mL}$. To create calibration curve, a series of work solutions $(0.05,0.1,0.2,0.3,0.4$, and $0.5 \mathrm{mg} / \mathrm{mL})$ were prepared by diluting the stock solution and filtered through a $0.2 \mu \mathrm{m}$ nylon cartridge just prior to HPLC analysis.

GRbE or individual ginsenoside $(\sim 20.0 \mathrm{mg})$ was accurately weighted into a $100.0 \mathrm{~mL}$ volumetric flask and dissolved in a mixture of acetonitrile/water (40:60) to make a final solution of $0.2 \mathrm{mg} / \mathrm{mL}$. The sample solution was filtered through a $0.2 \mu \mathrm{m}$ nylon cartridge before HPLC analysis.

\section{Validation of HPLC Method}

The HPLC method was validated for linearity and reproducibility. The linearity was investigated between peak area $(y)$ and the quantity $(x, \mu \mathrm{g})$ of each component. Five injections were performed to obtain the absorption plots ranging from 0.5 to $5.0 \mu \mathrm{g}$ in five increments. The precision and accuracy of the HPLC method were assessed by within and between run validations. The variation was evaluated by three injection of a mixture of five reference ginsenosides each day on three consecutive days after the preparation of reference ginsenoside solution. By substituting the peak area into the calibration curve equation from the same run, the measured concentrations were obtained. The coefficient of variance (RSD\%) was calculated by comparing the measured concentrations. The relative errors (RE\%) were obtained by comparing calculated and theoretical concentrations.

\section{HPLC Analysis}

The separation was carried out on a Hypersil HPLC ODS column $(250 \times 4.6 \mathrm{~mm}, 5 \mu \mathrm{m}$; detection: $203 \mathrm{~nm}$; flow rate: $1.5 \mathrm{~mL} / \mathrm{min}$; temperature: $25^{\circ} \mathrm{C}$ ). The water was employed as 
Table 1. Percentage of Total and Individual Ginsenosides $(\%$, Mean \pm s.d., $n=3)$

\begin{tabular}{|c|c|c|c|c|c|c|c|}
\hline Sample & $\mathbf{G}-\mathbf{R} \mathbf{b}_{1}$ & $\mathbf{G}-\mathbf{R c}$ & $\mathbf{G}-\mathbf{R} \mathbf{b}_{2}$ & $\mathbf{G}-\mathbf{R} \mathbf{b}_{3}$ & G-Rd & Total & 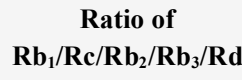 \\
\hline Sample A & $0.48 \pm 0.02$ & $0.07 \pm 0.01$ & $0.23 \pm 0.02$ & $0.68 \pm 0.03$ & $0.28 \pm 0.01$ & $1.75 \pm 0.02$ & $6.9 / 1.0 / 3.3 / 9.7 / 4.0$ \\
\hline GRbE A & $24.62 \pm 0.06$ & $3.89 \pm 0.02$ & $12.91 \pm 0.02$ & $37.25 \pm 0.04$ & $13.87 \pm 0.07$ & $92.54 \pm 0.12$ & 6.3/1.0/3.3/9.6/3.6 \\
\hline $\mathrm{GRbE} \mathrm{A}_{1}$ & $24.93 \pm 0.08$ & $3.68 \pm 0.06$ & $12.79 \pm 0.05$ & $37.16 \pm 0.07$ & $12.69 \pm 0.10$ & $91.25 \pm 0.36$ & $6.8 / 1.0 / 3.5 / 10.1 / 3.4$ \\
\hline Sample B & $0.49 \pm 0.03$ & $0.08 \pm 0.01$ & $0.26 \pm 0.01$ & $0.76 \pm 0.04$ & $0.27 \pm 0.02$ & $1.86 \pm 0.03$ & $6.1 / 1.0 / 3.3 / 9.5 / 3.4$ \\
\hline GRbE B & $23.86 \pm 0.08$ & $3.90 \pm 0.03$ & $12.68 \pm 0.03$ & $38.35 \pm 0.06$ & $13.16 \pm 0.05$ & $91.95 \pm 0.26$ & $6.1 / 1.0 / 3.3 / 9.8 / 3.4$ \\
\hline Sample C & $0.53 \pm 0.04$ & $0.03 \pm 0.01$ & $0.15 \pm 0.01$ & $0.28 \pm 0.06$ & $0.36 \pm 0.05$ & $1.35 \pm 0.05$ & $17.7 / 1.0 / 5.0 / 9.3 / 12.0$ \\
\hline GRbE C & $35.51 \pm 0.23$ & $2.08 \pm 0.05$ & $10.45 \pm 0.12$ & $18.43 \pm 0.17$ & $24.59 \pm 0.20$ & $91.06 \pm 0.19$ & 17.1/1.0/5.2/8.9/11.8 \\
\hline Sample E & $0.65 \pm 0.03$ & $0.04 \pm 0.01$ & $0.12 \pm 0.01$ & $0.34 \pm 0.02$ & $0.32 \pm 0.02$ & $1.47 \pm 0.02$ & $16.3 / 1.0 / 3.0 / 8.5 / 8.0$ \\
\hline Sample E & $39.05 \pm 0.11$ & $2.56 \pm 0.09$ & $7.89 \pm 0.16$ & $22.26 \pm 0.13$ & $19.83 \pm 0.11$ & $91.60 \pm 0.21$ & $15.3 / 1.0 / 3.1 / 8.7 / 7.7$ \\
\hline $\mathrm{G}-\mathrm{Rb}_{1}$ & $97.9 \pm 0.10$ & & & & & & \\
\hline $\mathrm{G}-\mathrm{Rb}_{3}$ & & & & $96.6 \pm 0.09$ & & & \\
\hline G-Rd & & & & & $98.6 \pm 0.08$ & & \\
\hline
\end{tabular}

mobile phase A, and acetonitrile was employed as mobile phase B. The gradient procedure is $0-36$ min with $31-37 \%$ B, 36-37 min with $37 \%$ B, 37-38 min with $37-90 \%$ B, $38-42$ min with $90 \% \mathrm{~B}, 42-43$ min with $90-31 \% \mathrm{~B}$, and $43-50$ min with $31 \% \mathrm{~B}$. The injection volume was $10 \mu \mathrm{L}$ for all analytes. Data collection and integration were performed using HP ChemStation software.

\section{RESULTS AND DISCUSSION}

\section{Preparation of Ginseng Rb Extract}

A standard method to generate the anticonvulsant ginseng $\mathrm{Rb}$ extract from the roots of American ginseng has been established in this study. This method allows preparation of large amounts of GRbE (about $10.0 \mathrm{~g}$ ) using column chromatography in two steps, first using an ODS column, followed by a silica gel column. It is expected that this method will produce GRbE in larger scale with commercial value when bigger chromatographic columns and larger amount of ODS or silica gel are applied.

The ability to reproducibly generate GRbE from same raw material will depend on the conditions of the extract, isolation and purification methods such as the ratio of raw material weight and solvent volume, the extraction time and temperature, the extract solvent, the ratio of loading sample and ODS or silica gel, and the eluting solvent. We used a standard method developed with the same conditions established in this study to prepare three batches of GRbEs A-A from the same resource of American ginseng (sample A). As shown in Fig. (2) and Table 1, GRbEs A- $\mathrm{A}_{2}$ enriched in five panaxadiol glycosides of $\mathrm{G}-\mathrm{Rb}_{1}, \mathrm{G}-\mathrm{Rc}, \mathrm{G}-\mathrm{Rb}_{2}, \mathrm{G}-\mathrm{Rb}_{3}$, and G-Rd with total ginsenosides of over $90 \%$, and had very similar ratio of $\mathrm{G}-\mathrm{Rb}_{1} / \mathrm{G}-\mathrm{Rc} / \mathrm{G}-\mathrm{Rb}_{2} / \mathrm{G}-\mathrm{Rb}_{3} / \mathrm{G}-\mathrm{Rd}$ as the raw material (sample A).

The ability to reproducibly generate GRbE will also depend, to a significant extent, on the raw material available. Previous study [12-14] and our analyses indicate that some ginseng products from different sources varied widely in content and relative proportions of ginsenosides. Therefore, the content and relative proportions of ginsenosides in GRbEs prepared from different sources of ginseng products may be different. Besides the three batches of GRbEs A- $\mathrm{A}_{2}$ reproducibly prepared from sample $A$, we had generated additional four batches of GRbEs B-E from different sources (samples B-E) using the same method for the preparation of GRbEs A-A $A_{2}$. As shown in Table 1, GRbEs B-E also contained five panaxadiol glycosides $\left(\mathrm{G}-\mathrm{Rb}_{1}, \mathrm{G}-\mathrm{Rc}\right.$, G$\mathrm{Rb}_{2}, \mathrm{G}-\mathrm{Rb}_{3}$, and G-Rd) with total ginsenosides of over $90 \%$ as GRbEs $A-A_{2}$, but the ratios of $G-R b_{1} / G-R c / G-R b_{2} / G-$ $\mathrm{Rb}_{3} / \mathrm{G}-\mathrm{Rd}$ in GRbEs B-E and GRbEs $\mathrm{A}-\mathrm{A}_{2}$ are different. This difference is ascribed to the different ratios of $\mathrm{G}-\mathrm{Rb}_{1} / \mathrm{G}$ $\mathrm{Rc} / \mathrm{G}-\mathrm{Rb}_{2} / \mathrm{G}-\mathrm{Rb}_{3} / \mathrm{G}-\mathrm{Rd}$ in samples A-E (Table $\mathbf{1}$ ).

\section{Isolation and Purification of Individual Ginsenosides $R b_{1}$, $R b_{3}$, and $R d$ in Large Scale}

High-speed counter-current chromatography (HSCCC) has been successfully utilized for preparative isolation of G$\mathrm{Rb}_{1}[15,16]$ and G-Rd [15] from ginseng plants. HSCCC has the advantage of mild separation condition with a $100 \%$ recovery of the load sample without the solid-phase materials, but only a small amount (milligram level) of individual ginsenoside can be achieved $[15,16]$. In this study, a simple, rapid and convenient column chromatographic method has been used and enable to purify three ginsenosides of $\mathrm{G}-\mathrm{Rb}_{1}$ 

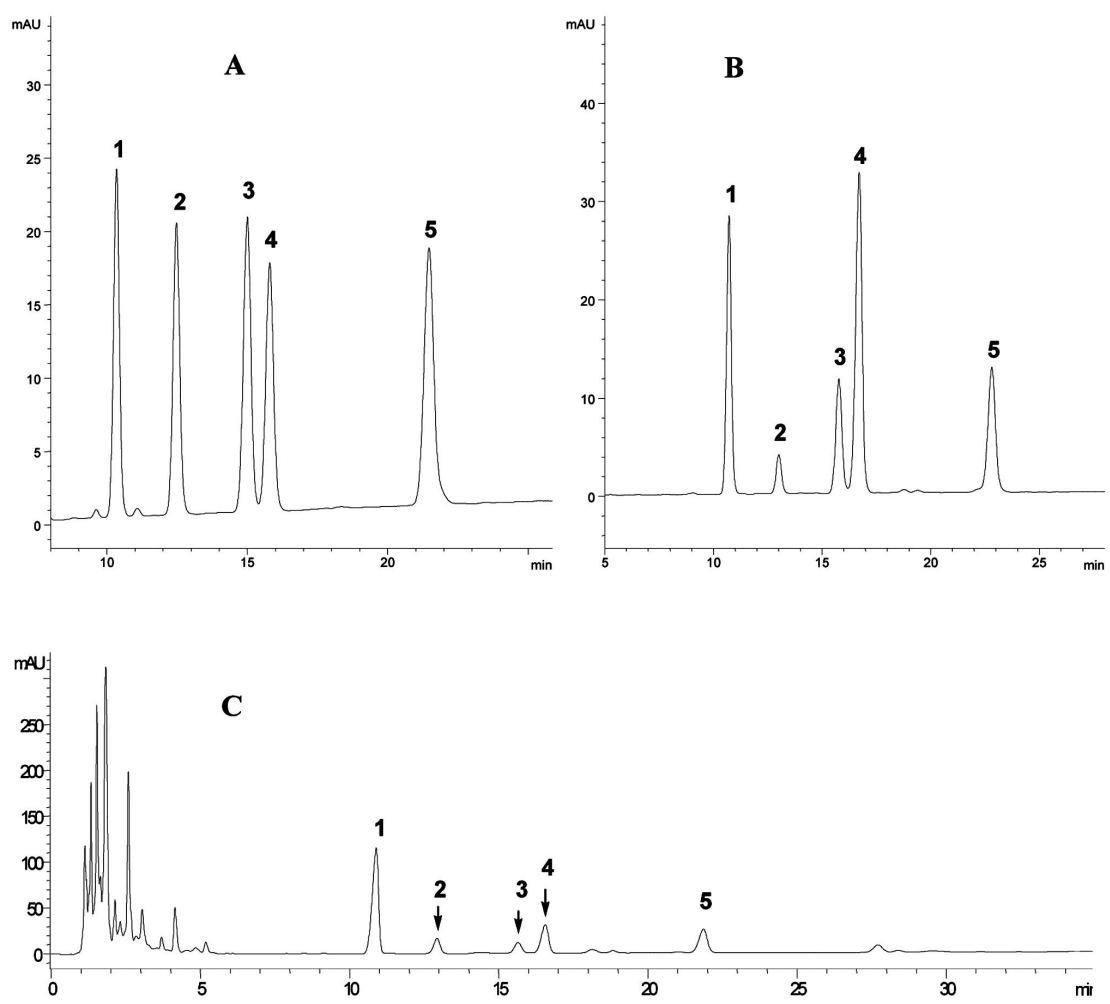

Fig. (2). HPLC chromatograms of reference ginsenosides (A), GRbE A (B), and sample B (C) (1. GRb1, 2. G-Rc, 3. G-Rb2, 4. G-Rb3, 5. G-Rd).

(4.12 g), G-Rb $3(3.56 \mathrm{~g})$ and G-Rd (2.05 g) from GRbE using an ODS column $(600 \times 70 \mathrm{~mm})$. The current work demonstrates that this method can generate large amounts (gram level) of these three compounds, enough for bioactivity studies, analytic purposes and other uses. Furthermore, the ODS column used to generate these three ginsenosides can be repeatedly used to reduce the cost.

\section{HPLC Method Validation}

The HPLC method was validated for linearity and reproducibility. The linearity of the standard curves was studied for $\mathrm{G}-\mathrm{Rb}_{1}, \mathrm{G}-\mathrm{Rb_{2 }}, \mathrm{G}-\mathrm{Rb}_{3}, \mathrm{G}-\mathrm{Rc}$, and G-Rd. The standard curve of each ginsenoside was investigated between the peak area $(y)$ and the quantity of each ginsenoside $(x, \mu \mathrm{g})$. The linearity is expressed in terms of the correlation coefficient $(\gamma)$. The correlation coefficient was found to be better than 0.9999 for each ginsenoside in the range of 0.5 to $5.0 \mu \mathrm{g}$.

The reproducibility of the method was assessed by within and between run validations. The $\mathrm{RSD} \%$ and $\mathrm{RE} \%$ were found to be less than $1.41 \%$ and $1.30 \%$, respectively.

\section{Chemical Profiles of Ginseng Rb Extract (GRbE)}

The chemical profile of GRbE was characterized by defining the chemical constituents and measuring the concentration of total and individual ginsenosides using HPLC. Five compounds were isolated and purified from GRbE by preparative and analytic HPLC. The structures (Fig. 1) of these compounds were determined by extensive NMR spec-

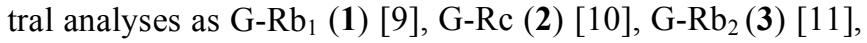
$\mathrm{G}^{-\mathrm{Rb}_{3}}$ (4) [10], and G-Rd (5) [9]. Compounds 1-5 had purity of $98.5 \%-99.3 \%$ determined by HPLC and were used as reference compounds.
The established HPLC method was used to determine the chemical constituents and quantitate the concentration of total and individual ginsenosides of GRbEs A-E. As shown in Fig. (2) and Table 1, the seven batches of GRbEs A-E prepared in this study contain the same chemical constituents: $G-R_{1}\left(t_{R}: 10.7 \mathrm{~min}\right), G-R c\left(t_{R}: 13.0 \mathrm{~min}\right), G-\mathrm{Rb}_{2}\left(\mathrm{t}_{\mathrm{R}}\right.$ : $15.8 \mathrm{~min}), \mathrm{G}-\mathrm{Rb}_{3}\left(\mathrm{t}_{\mathrm{R}}: 16.7 \mathrm{~min}\right)$, and $\mathrm{G}-\mathrm{Rd}\left(\mathrm{t}_{\mathrm{R}}: 22.8 \mathrm{~min}\right)$, and the total ginsenosides for each individual preparations is over $90 \%$.

\section{CONCLUSION}

A standardized method to prepare the anticonvulsant GRbE from different American ginseng resources by a combination of ODS and silica gel open column chromatography has been established. The structures of the individual ginsenosides from GRbE were confirmed by extensive NMR analysis. A validated HPLC method was used to verify the chemical constituents and to determine the concentration of total and individual ginsenosides of GRbE. This study demonstrates the application of the established methods for reproducibly generating a standardized anticonvulsant GRbE. This active GRbE concentrated five panaxadiol ginsenosides of $\mathrm{Rb}_{1}, \mathrm{Rb}_{2}, \mathrm{Rb}_{3}, \mathrm{Rc}$, and $\mathrm{Rd}$ with total ginsenosides over $90 \%$, and meet the requirement for an ongoing preclinical investigation on its potential for the treatment of epilepsy.

\section{ACKNOWLEDGEMENTS}

This study was partially supported by a grant from The Epilepsy Research Foundation to Dr. Janet L. Stringer of Baylor College of Medicine in Houston, Texas and a grant from Science and Technology Commission of Shanghai $\mathrm{Mu}-$ nicipality to Xiao-Yuan Lian. The authors would like to thank the Keck/IMD NMR Center, which is funded by the 
W. M. Keck Foundation and the University of Houston, and Dr. Youlin Xia for NMR analysis assistance.

\section{ABBREVIATIONS}

\begin{tabular}{|c|c|}
\hline $\mathrm{G}-\mathrm{Rb}_{1}$ & $=$ Ginsenoside $\mathrm{Rb}_{1}$ \\
\hline $\mathrm{G}-\mathrm{Rb}_{2}$ & $=$ Ginsenoside $\mathrm{Rb}_{2}$ \\
\hline $\mathrm{G}-\mathrm{Rb}_{3}$ & $=$ Ginsenoside $\mathrm{Rb}_{3}$ \\
\hline G-Rc & $=$ Ginsenoside $\mathrm{Rc}$ \\
\hline G-Rd & $=$ Ginsenoside $\mathrm{Rd}$ \\
\hline $\mathrm{GRbE}$ & $=$ Ginseng $\mathrm{Rb}$ extract \\
\hline HSCCC & $\begin{aligned}= & \text { High-speed counter-current } \\
& \text { phy }\end{aligned}$ \\
\hline
\end{tabular}

\section{REFERENCES}

[1] Kuhn, M.A.; Winston, D. Herbal therapy and supplements: a scientific and traditional approach. Lippincott: New York, 2000.

[2] Attele, A.S.; Wu, J.A.; Yuan, C.S. Ginseng pharmacology: multiple constituents and multiple actions. Biochem. Pharmacol., 1999, 58(11), 1685-1693.

[3] Zhu, S.; Zou, K.; Cai, S.Q.; Meselhy, M.R.; Komatsu, K. Simultaneous determination of triterpene saponins in ginseng drugs by high-performance liquid chromatography. Chem. Pharm. Bull., 2004, 52(8), 995-998.

[4] Banskota, A.H.; Tezuka, Y.; Le Tran, Q.; Kadota, S. Chemical constituents and biological activities of Vietnamese medicinal plants. Curr. Top. Med. Chem., 2003, 3(2), 227-248.

[5] Zou, K.; Zhu, S.; Meselhy, M. R.; Tohda, C.; Cai, S.Q.; Komatsu K. Dammarane-type saponins from Panax japonicus and their neurite outgrowth activity in SK-N-SH cell. J. Nat. Prod., 2002, 65(9), 1288-1292.
[6] Lian, X.Y.; Zhang, Z.Z.; Stringer, J.L. Anticonvulsant activity of ginseng on seizures induced by chemical convulsants. Epilepsia, 2005, 46(1), 15-22.

[7] Lian, X.Y.; Zhang, Z.Z.; Stringer, J.L. Anticonvulsant and neuroprotective effects of ginsenosides in rats. Epilepsy Res., 2006, 70(23), 244-256.

[8] Lian, X.Y.; Zhang, Z.Z.; Stringer, J.L. Protective effects of ginseng components in a rodent model of neurodegeneration. Ann. Neurol., 2005, 57(5), 642-648.

[9] Dong, A.; Ye, M. Guo, H.; Zheng, J.; Guo, D. Microbial transformation of ginsenoside $\mathrm{Rb}_{1}$ by Rhizopus stolonifer and Curvularia lunata. Biotechnol. Lett., 2003, 25(4), 339-344.

[10] Liu, C., Han, J., Quan, Y.; Huang, X.; Wang, H. Purification and quantification of ginsenosides $\mathrm{Rb}_{3}$ and $\mathrm{Rc}$ from crude extracts of caudexes and leaves of Panax notoginseng. Sep. Purif. Technol., 2007, 54, 198-203.

[11] Tanaka, O.; Kasai, R. In: Herz, W.; Grisebach, H.; Kirby, G.W.; Tamm C, Eds. Progress in the Chemistry of Organic Natural Products. Springer-Verlag, Wien-New York, 1984, Vol. 46, pp. 1-76.

[12] Cui, J.; Garle, M.; Eneroth, P.; Björkhem, I. What do commercial ginseng preparations contain? Lancet, 1994, 344(8915), 134.

[13] Harkey, M.R.; Henderson, G.L.; Gershwin, M.E.; Stern, J.S.; Hackman, R.M. Variability in commercial ginseng products: an analysis of 25 preparations. Am. J. Clin. Nutr., 2001, 73(6), 11011106.

[14] Li, W.; Fitzloff, J.F. HPLC determination of ginsenosides content in ginseng dietary supplements using ultraviolet detection. J. Liq. Chromatogr. R. T., 2002, 25(16), 2485-2500.

[15] Cao, X.L.; Tian, Y.; Zhang, T.Y.; Liu, Q.H.; Jia, L.J.; Ito, Y. Separation of dammarane-saponins from notoginseng, root of Panax notoginseng (Burk.) F.H. Chen, by HSCCC coupled with evaporative light scattering detector. J. Liq. Chromatogr. R. T., 2003, 26(9-10), 1579-1591.

[16] Du, Q.; Jerz, G.; Waibel, R.; Winterhalter, P. Isolation of dammarane saponins from Panax notoginseng by high-speed countercurrent chromatography. J. Chromatogr. A., 2003, 1008(2), 173180.

(C) Zhang and Lian; Licensee Bentham Open.

This is an open access article licensed under the terms of the Creative Commons Attribution Non-Commercial License (http://creativecommons.org/ licenses/ by-nc/3.0/) which permits unrestricted, non-commercial use, distribution and reproduction in any medium, provided the work is properly cited. 